\title{
ON CERTAIN CLASSES OF MEROMORPHICALLY STARLIKE FUNCTIONS
}

\author{
NAK EUN CHO and JI A. KIM \\ Department of Applied Mathematics \\ National Fisheries University of Pusan \\ Pusan 608-737, Korea \\ (Received May 11, 1993 and in revised form April 14, 1994)
}

\begin{abstract}
The object of the present paper is to introduce a new class $\Sigma_{n}(\alpha)$ of meromorphic functions defined by a multiplier transformation and to investigate some properties for the class $\Sigma_{n}(\alpha)$. Further we study integrals of functions in $\Sigma_{n}(\alpha)$.
\end{abstract}

KEY WORDS AND PHRASES. Univalent functions, meromorphically starlike functions, integral operators.

1991 AMS SUBJECT CLASSIFICATION CODE. 30C45.

\section{INTRODUCTION.}

Let $\Sigma$ denote the class of functions of the form

$$
f(z)=\frac{a_{-1}}{z}+\sum_{k=0}^{\infty} a_{k} z^{k}\left(a_{-1} \neq 0\right)
$$

which are regular in the punctured disk $D=\{z: 0<|z|<1\}$. For any integer $n$, let the operator $I^{n}$ operating on $f \in \Sigma$ be defined by

$$
I^{n} f(z)=\frac{a_{-1}}{z}+\sum_{k=0}^{\infty}(k+2)^{-n} a_{k} z^{k}
$$

Obviously, we have

$$
I^{n}\left(I^{m} f(z)\right)=I^{n+m} f(z)
$$

for all integers $m$ and $n$. For any nonpositive integer $n$, the operators $I^{n}$ are the differential operators studied by Uralegaddi and Somanatha [5]. Also the operators $I^{n}$ are closely related to the multiplier transformations introduced by Flett [2].

For any integer $n$, let $\Sigma_{n}(\alpha)$ denote the class of functions $f \in \Sigma$ satisfying the condition

$$
\operatorname{Re}\left\{\frac{I^{n-1}}{I^{n} f(z)}-2\right\}<-\alpha(0 \leq \alpha<1, z \in U=\{z:|z|<1\}) .
$$

In this paper, we prove that for the classes $\Sigma_{n}(\alpha)$ of functions in $\Sigma, \Sigma_{n}(\alpha) \subset \Sigma_{n+1}(\alpha)$ holds. Since $\Sigma_{0}(\alpha)$ equals $\Sigma^{*}(\alpha)$ (the class of meromorphically starlike functions of order $\alpha$ ), all members in $\Sigma_{n}(\alpha)$ are univalent for any nonpositive integer $n$. Further property preserving integrals are considered Our results generalize the some results of Bajpai [1], Goel and Sohi [3] and Uralegaddi and Somanatha [6]. 


\section{MAIN RESULTS.}

We begin with the statement of the following lemma due to Miller and Mocaun [4].

LEMMA. Let $\phi(u, v)$ be a complex valued function, $\phi: D \rightarrow C, D \subset C^{2} \in(C$ is the complex plane), and let $u=u_{1}+i u_{2}, v=v_{1}+i v_{2}$. Suppose that the function $\phi(u, v)$ satisfies the following condition

(i) $\phi(u, v)$ is continuous in $D$,

(ii) $(1,0) \in D$ and $\operatorname{Re}\{\phi(1,0)\}>0$;

(iii) $\operatorname{Re}\left\{\phi\left(i u_{2}, v_{1}\right)\right\} \leq 0$ for all $\left(i u_{2}, v_{1}\right) \in D$ such that $v_{1} \leq \frac{-\left(1+u_{2}^{2}\right)}{2}$.

Let $p(z)=1+p_{1} z+p_{2} z^{2}+\cdots$ be regular in $U$ such that $\left(p(z), z p^{\prime}(z)\right) \in D$ for all $z \in U$. If

$$
\operatorname{Re}\left\{\phi\left(p(z), z p^{\prime}(z)\right)\right\}>0 \quad(z \in U),
$$

then $\operatorname{Re}\{p(z)\}>0(z \in U)$.

With the aid of above lemma, we drive

THEOREM 1. If $f \in \Sigma_{n}(\alpha)$, then $f \in \Sigma_{n+1}(\beta)$, where

$$
\beta=\frac{5+2 \alpha-\sqrt{(3-2 \alpha)^{2}+8}}{4} .
$$

PROOF. Define the function $p(z)$ by

$$
\frac{I^{n} f(z)}{\Gamma^{n+1} f(z)}=\gamma+(1-\gamma) p(z)
$$

where

$$
\gamma=\frac{(3-2 \alpha)+\sqrt{(3-2 \alpha)^{2+8}}}{4}(\gamma>1) .
$$

We see that $p(z)=1+p_{1} z+p_{2} z^{2}+\cdots$ is regular in $U$. Making use of the logarithmic differentiations of both sides in (2.2) and using the identity

$$
z\left(I^{n} f(z)\right)^{\prime}=I^{n-1} f(z)-2 I^{n} f(z)
$$

we obtain

$$
\frac{I^{n-1} f(z)}{I^{n} f(z)}=\gamma+(1-\gamma) p(z)+\frac{(1-\gamma) z p^{\prime}(z)}{\gamma+(1-\gamma) p(z)}
$$

or

$$
-\operatorname{Re}\left\{\frac{I^{n-1} f(z)}{I^{n} f(z)}-2+\alpha\right\}=\operatorname{Re}\left\{2-\left(\alpha+\gamma-\left(1-\gamma p(z)-\frac{(1-\gamma) z p^{\prime}(z)}{\gamma+(1-\gamma p(z)}\right\}>0 .\right.\right.
$$

Let us define the function $\phi(u, v)$ by

$$
\phi(u, v)=2-(\alpha+\gamma)-(1-\gamma) u-\frac{(1-\gamma) v}{\gamma+(1-\gamma) u}
$$

Then $\phi(u, v)$ satisfies

(i) $\phi(u, v)$ is continuous in $D=\left(C-\left\{\frac{\gamma}{\gamma-1}\right\}\right) \times C$;

(ii) $(1,0) \in D$ and $\operatorname{Re}\{\phi(1,0)\}=1-\alpha>0$; 
(iii) for all $\left(i u_{2}, v_{1}\right) \in D$ such that $v_{1} \leq \frac{-\left(1+u_{2}^{2}\right)}{2}$,

$$
\begin{aligned}
\operatorname{Re}\left\{\phi\left(i u_{2}, v_{1}\right)\right\} & =2-(\alpha+\gamma)-\frac{\gamma(1-\gamma) v_{1}}{\gamma^{2}+(1-\gamma)^{2} u_{2}^{2}} \\
& \leq 2-(\alpha+\gamma)+\frac{\gamma(1-\gamma) v_{2}}{2\left(\gamma+(1-\gamma)^{2} u_{2}^{2}\right)} \\
& \leq 0 .
\end{aligned}
$$

Thus the function $\phi(u, v)$ satisfies the conditions in our Lemma This shows that $\operatorname{Re}\{p(z)\}>0$ $(z \in U)$, hence

$$
\operatorname{Re}\left\{\frac{I^{n} f(z)}{I^{n+1} f(z)}\right\}<\gamma(z \in U)
$$

or

$$
\operatorname{Re}\left\{\frac{I^{n} f(z)}{I^{n+1} f(z)}-2\right\}<-\beta(z \in U)
$$

where $\beta$ is given by (21) Therefore we complete the proof of the theorem

Since $\beta-\alpha>0$ in Theorem 1, we have

COROLLARY 1. $\Sigma_{n}(\alpha) \subset \Sigma_{n+1}(\alpha)$ for any integer $n$

REMARK. For nonpositive integers $n$, Corollary 1 is a similar result obtained by Uralegaddi and Somanatha [6]

Putting $n=0$ and $\alpha=0$ in Corollary 1, we obtain the following result of Bajpai [1]

COROLLARY 2. If $f(z)=\frac{a_{1}}{z}+\sum_{k=0}^{\infty} a_{k} z^{k}\left(a_{-1} \neq 0\right)$ is meromorphically starlike, then so is

$$
F_{1}(z)=\frac{1}{x^{2}} \int_{0}^{z} t f(t) d t
$$

Next, we prove

CHEOREM 2. Let $f \in \Sigma_{n}(\alpha)$ and let

$$
F_{c}(z)=\frac{c}{z^{c+1}} \int_{0}^{z} t^{c} f(t) d t(c>0)
$$

Then $F_{c} \in \Sigma_{n}(\beta)$, where

$$
\beta=\frac{(3+2 a c)-\sqrt{(5-2 \alpha-2 c)^{2}+8(4 c-3-2 \alpha c+2 \alpha)}}{4}
$$

PROOF. Let $f \in \Sigma_{n}(\alpha)$. Then we have

$$
\operatorname{Re}\left\{\frac{I^{n-1} f(z)}{I^{n} f(z)-2}\right\}<-a .
$$

From the definition of $F_{c}$, we obtain

$$
z\left(I^{n} F_{c}(z)\right)^{\prime}=c I^{n} f(z)-(c+1) I^{n} F_{c}(z)
$$

and also 


$$
z\left(I^{n} F_{c}(z)\right)^{\prime}=I^{n-1} F_{c}(z)-2 I^{n} F_{c}(z)
$$

Using (2.14) and (2 15), the condition (2 13) may be written as

$$
\operatorname{Re}\left\{\frac{\frac{I^{n-2} F_{c(z)}}{I^{n-1} F_{c}(z)}+(c-1)}{1+(c-1) \frac{I^{n} F_{c}(z)}{I^{n-1} F_{c}(z)}}-2\right\}<-\alpha
$$

Define the function $p(z)$ by

$$
\frac{I^{n-1} F_{c}(z)}{I^{n} F_{c}(z)}=\gamma+(1-\gamma) p(z)
$$

where

$$
\gamma=\frac{(5-2 \alpha-2 c)+\sqrt{(5-2 \alpha-2 c)^{2}+8(4 c-3-2 \alpha c+2 \alpha)}}{4}(\gamma>1) .
$$

Then $p(z)=1+p_{1} z+p_{2} z^{2}+\cdots$ is regular in $U$. Differentiating (2.17) logarithmically and simplifying, we have

$$
\frac{\frac{I^{n-2} F_{c}(z)}{I^{n-1} F_{c}(z)}+(c-1)}{1+(c-1) \frac{I^{n} F_{c}(z)}{I^{n-1} F_{c}(z)}}-2=-2+\gamma+(1-\gamma) p(z)+\frac{\left(1-\gamma z p^{\prime}(z)\right.}{(\gamma+c-1)+(1-\gamma p(z)}
$$

It follows from (2.19) that

$$
\begin{aligned}
& -\operatorname{Re}\left\{\frac{\frac{I^{n-2} F_{c}(z)}{I^{n-1} F_{c}(z)}+(c-1)}{1+(c-1) \frac{I^{n} F_{c}(z)}{I^{n-1} F_{c}(z)}}-2+\alpha\right\} \\
& =\operatorname{Re}\left\{2-(\alpha+\gamma)-(1-\gamma) p(z)-\frac{(1-\gamma) z p^{\prime}(z)}{(\gamma+c-1)+(1-\gamma) p(z)}\right\} \\
& >0 .
\end{aligned}
$$

If we define the function $\phi(u, v)$ by

$$
\phi(u, v)=2-(\alpha+\gamma)-(1-\gamma) u-\frac{(1-\gamma) v}{(\gamma+c-1)+(1-\gamma) u},
$$

then $\phi(u, v)$ satisfies

(i) $\phi(u, v)$ is continuous in $D=\left(C-\left\{\frac{\gamma+c-1}{\gamma-1}\right\}\right) \times C$;

(ii) $(1,0) \in D$ and $\operatorname{Re}\{\phi(1,0)\}=1-\alpha>0$;

(iii) for all $\left(i u_{2}, v_{1}\right) \in D$ such that $v_{1} \leq \frac{-\left(1+u_{2}^{2}\right)}{2}$,

$$
\begin{aligned}
\operatorname{Re}\left\{\phi\left(i u_{2}, v_{1}\right)\right\} & =2-\left(\alpha+\gamma-\frac{(\gamma+c-1)(1-\gamma) v_{1}}{(\gamma+c-1)^{2}+(1-\gamma)^{2} u_{2}^{2}}\right. \\
& \leq 2-(\alpha+\gamma)+\frac{(\gamma+c-1)(1-\gamma)\left(1+u_{2}^{2}\right)}{2\left\{(\gamma+c-1)^{2}+(1-\gamma)^{2 u_{2}^{2}}\right\}} \\
& \leq 0
\end{aligned}
$$


Since $\phi(u, v)$ satisfies the conditions in Lemma, we have that $\operatorname{Re}\{p(z)\}>0(z \in U)$. This proves that

$$
\operatorname{Re}\left\{\frac{I^{n-1} F_{c}(z)}{I^{n} F_{c}(z)}\right\}<\gamma(z \in U)
$$

or

$$
\operatorname{Re}\left\{\frac{I^{n-1} F_{c}(z)}{I^{n} F_{c}(z)}-2\right\}<-\beta(z \in U),
$$

where $\beta$ is given by (2 12). That is, $F_{c}(z) \in \Sigma_{n}(\beta)$.

Similarly, from Theorem 2, we have

COROLLARY 3. If $f \in \Sigma_{n}(\alpha)$, then the integral operator $F_{c}$ defined by (2.11) belongs to the class $\Sigma_{n}(\alpha)$.

Taking $n=0$ and $\alpha=0$ in Corollary 3, we obtained the following corresponding result of Goel and Sohi [3].

COROLLARY 4. If $f(z)=\frac{a_{-1}}{z}+\sum_{k=0}^{\infty} a_{k} z^{k}$ is meromorphically starlike, then so is the integral operator $F_{c}$ defined by (2.11).

The following theorem gives us a characterization of the class $\Sigma_{n}(\alpha)$.

THEOREM 3. $f \in \Sigma_{n}(\alpha)$ if and only if the integral operator $F_{1}$ defined by (2.10) belongs to the class $\Sigma_{n-1}(\alpha)$.

PROOF. For $c=1$, the identities (2.14) and (2.15) reduce to $I^{n} f(z)=I^{n-1} F_{1}(z)$ and hence $I^{n-1} f(z)=I^{n-2} F_{1}(z)$. Therefore

$$
\frac{I^{n-1} f(z)}{I^{n} f(z)}=\frac{I^{n-2} F_{1}(z)}{I^{n-1} F_{1}(z)}
$$

and the result follows.

ACKNOWLEDGEMENT. This paper was supported (in part) by Non-Directed Research Fund and the Research Institute Attached to University Program, Korea Research Foundation, 1993.

\section{REFERENCES}

1. BAJPAI, S.K., A note on a class of meromorphic univalent functions, Rev. Roumanie Math. Pure Appl. 22 (1977), 295-297.

2. FLETT, T.M., The dual of an inequality of Hardy and Littlewood and some related inequalities, J. Math. Anal. Appl. 38 (1972), 746-765.

3. GOEL, R.M. and SOHI, N.S., On a class of meromorphic functions, Glas. Mat., 17 (1981), 19-28.

4. MILLER, S.S. and MOCANU, P.T., Second order differential inequalities in the complex plane, J. Math. Anal. Appl. 65 (1978), 289-305.

5. URALEGADDI, B.A. and SOMANATHA, C., Certain differential operators for meromorphic functions, Houston J. Math., 17 (1991), 279-284.

6. URALEGADDI, B.A. and SOMANATHA, C., New criteria for meromorphic starlike univalent functions, Bull. Aust. Math. Soc., 43 (1991), 137-140. 


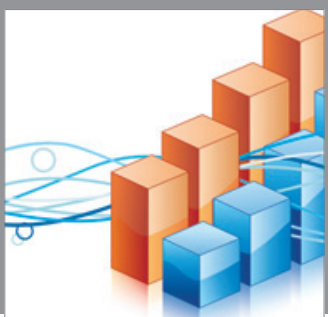

Advances in

Operations Research

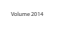

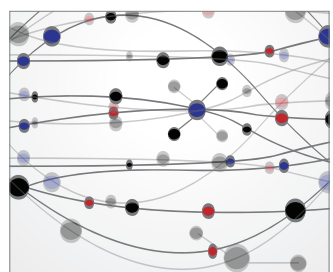

\section{The Scientific} World Journal
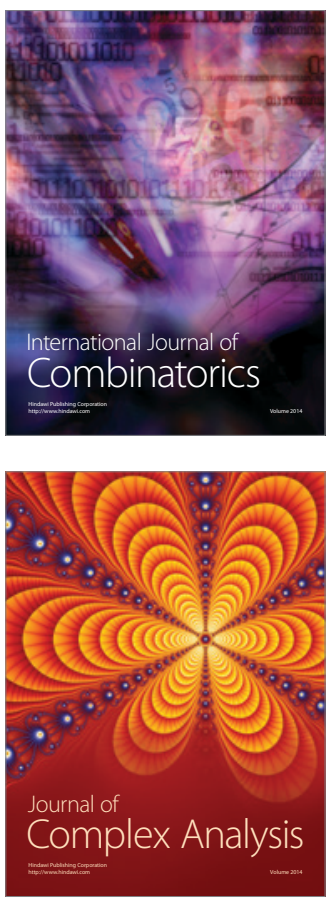

International Journal of

Mathematics and

Mathematical

Sciences
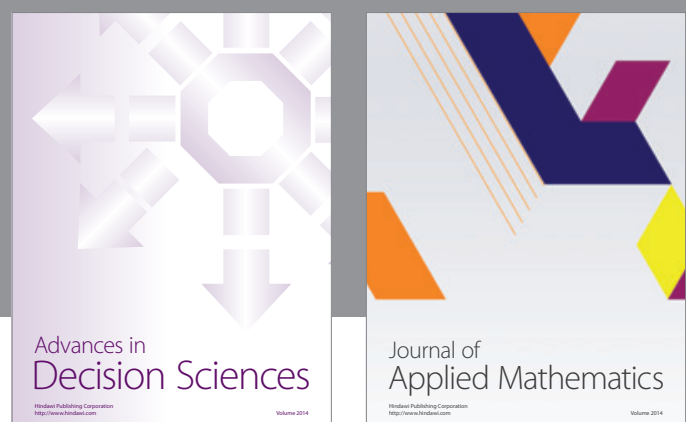

Journal of

Applied Mathematics
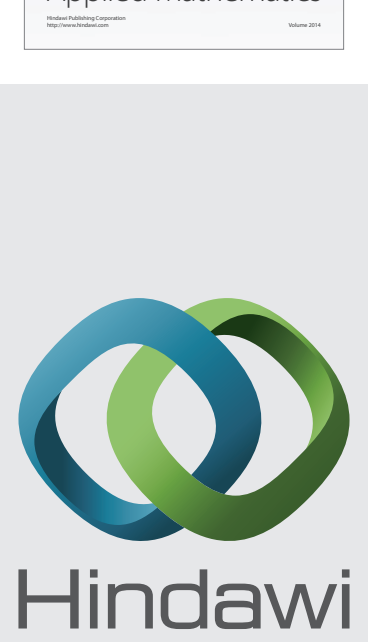

Submit your manuscripts at http://www.hindawi.com
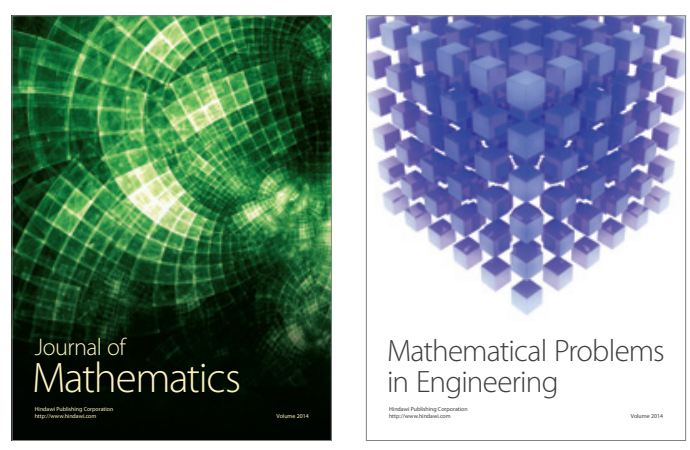

Mathematical Problems in Engineering
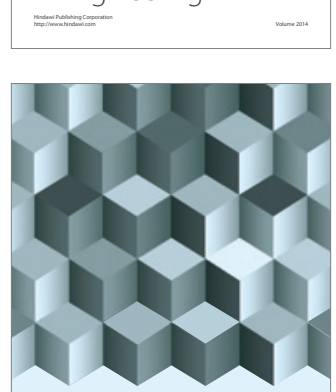

Journal of

Function Spaces
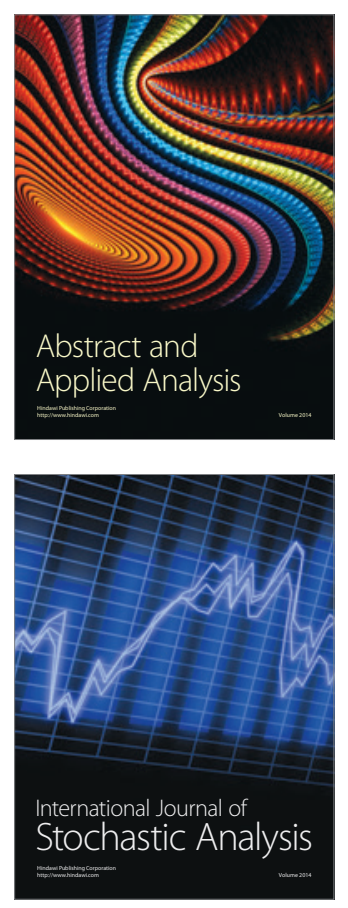

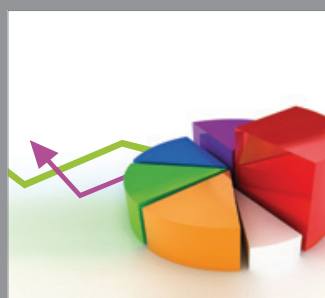

ournal of

Probability and Statistics

Promensencen
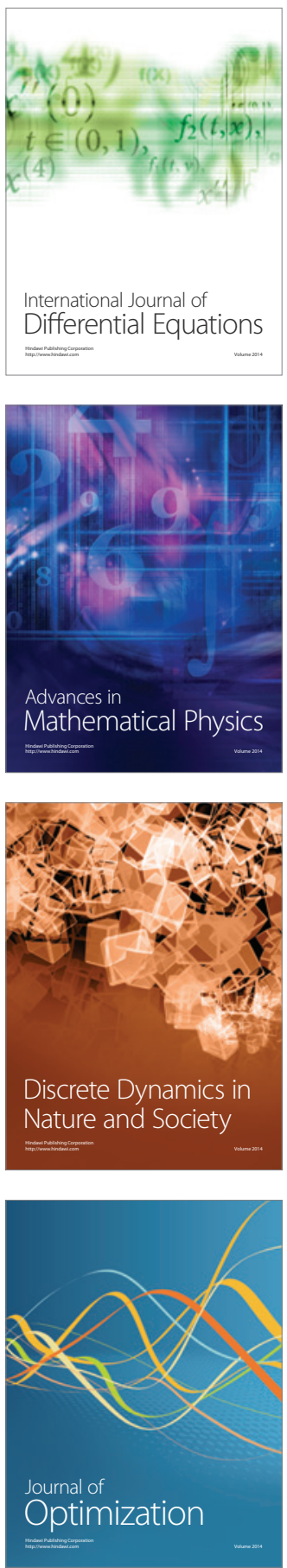